\title{
Norois
}

Environnement, aménagement, société

193 | 2004/4

Varia

\section{Hiérarchisation des facteurs structurant les dynamiques pluriannuelles des sols nus hivernaux}

\author{
Application au bassin versant du Yar (Bretagne)
}

Driving factors for pluri-annual changes of winters bare soils. A hierarchization in the Yar watershed area, Brittany

\section{Samuel Corgne}

\section{OpenEdition}

\section{Journals}

Édition électronique

URL : https://journals.openedition.org/norois/713

DOI : $10.4000 /$ norois.713

ISBN : 978-2-7535-1541-3

ISSN : 1760-8546

\section{Éditeur}

Presses universitaires de Rennes

\section{Édition imprimée}

Date de publication : 1 décembre 2004

Pagination : 17-29

ISBN : 978-2-7535-0081-5

ISSN : 0029-182X

\section{Référence électronique}

Samuel Corgne, « Hiérarchisation des facteurs structurant les dynamiques pluriannuelles des sols nus hivernaux », Norois [En ligne], 193 | 2004/4, mis en ligne le 14 août 2008, consulté le 13 janvier 2022. URL : http://journals.openedition.org/norois/713 ; DOI : https://doi.org/10.4000/norois.713

Ce document a été généré automatiquement le 13 janvier 2022.

(C) Tous droits réservés 


\title{
Hiérarchisation des facteurs structurant les dynamiques pluriannuelles des sols nus hivernaux
}

\author{
Application au bassin versant du Yar (Bretagne) \\ Driving factors for pluri-annual changes of winters bare soils. A hierarchization \\ in the Yar watershed area, Brittany
}

\section{Samuel Corgne}

\section{NOTE DE L'ÉDITEUR}

Cet article a été reçu le 10 novembre 2003 et définitivement accepté le 3 juin 2004.

Remerciements : Ce travail est financé par le conseil régional de Bretagne. Je remercie Stéphanie Martinez et Denis Rousset de la chambre d'agriculture des Côtes-d'Armor, Bénédicte Le Bref de la communauté de communes de Lannion pour leur aide dans l'identification des facteurs de changement. Je remercie également Claudine Thenail (INRA) pour le temps consacré à m'expliquer l'information mutuelle entre les variables ainsi que Laurence Hubert-Moy (Maître de conférence à l'Université de Rennes 2) et Bernard Clément (Maître de conférence à l'Université de Rennes 1) pour leurs précieux conseils méthodologiques.

\section{Introduction}

1 La Bretagne connaît depuis plusieurs décennies des changements de systèmes culturaux, liés à des modifications des structures paysagères et des pratiques agricoles entraînant des problèmes environnementaux, en particulier une dégradation importante des ressources en eau (Wicherek, 1996). La mise en place depuis le début 
des années 1990 de programmes de restauration de la qualité de l'eau (Bretagne Eau Pure, PMPOA - Programme de Maîtrise des Pollutions d'Origine Agricole -) a enclenché des modifications des systèmes agraires dont certaines peuvent se traduire spatialement. Dans ce contexte, la connaissance des variations spatio-temporelles de l'utilisation et de l'occupation des sols constitue un élément-clé dans une démarche de restauration de la qualité de l'eau. Parmi les différents types d'occupation du sol, les successions d'états de couverts végétaux sur les parcelles en hiver influent particulièrement sur les transferts de flux polluants, jouant comme un accélérateur lorsque les parcelles sont laissées nues après des cultures telles que le maïs ou les céréales, ou comme un frein lorsqu'elles sont couvertes par des intercultures (Dabney, 1998 ; Jaunatre, 1997).

Le suivi du changement hivernal de l'occupation des sols peut être effectué à partir de données de télédétection. Les images à haute résolution de type Landsat TM, SPOT ou radar, d'une précision décamétrique, permettent ainsi de réaliser des cartographies des successions culturales à l'échelle de la parcelle, l'analyse des changements étant produite à l'échelle du bassin versant (Hubert-Moy et al., 2001). À cette échelle infrarégionale, correspondant à des secteurs dont la superficie varie en général d'une dizaine de $\mathrm{km}^{2}$ à plusieurs centaines de $\mathrm{km}^{2}$, les images permettent d'effectuer, à l'aide de données d'origines diverses (socio-économiques, fonctionnement physique du milieu, etc.) des analyses multitemporelles des changements observés.

3 L'identification des facteurs de changement de l'occupation hivernale du sol dans les régions agricoles intensives représente un enjeu important dans la compréhension des dynamiques spatiales ayant un impact significatif sur l'environnement et le paysage. Elle permet d'envisager la construction de scénarii prédictifs (Hubert-Moy et al., 2002). Ce travail de modélisation pose cependant un certain nombre de problèmes méthodologiques. Dans un premier temps interviennent la détermination de la nature, de l'origine des changements détectés et du choix des variables permettant de décrire les changements observés, tandis que l'identification et la hiérarchisation des facteurs de changements observés apparaissent ensuite comme un élément-clé de la mise en œuvre du processus de simulation de l'évolution de l'occupation et de l'utilisation des sols.

4 L'objectif de ce travail est de définir le poids respectif des facteurs de changements identifiés sur un bassin versant localisé en secteur agricole assez intensif faisant l'objet de programmes de restauration de la qualité de l'eau et sur lequel une détection des changements de la couverture hivernale des sols a déjà été réalisée (Corgne et al., 2002).

5 Pour cela, après avoir brièvement décrit la problématique de l'identification et de la caractérisation des facteurs de changement concernant la couverture hivernale des sols en contexte agricole intensif, la démarche de hiérarchisation des facteurs de changements identifiés sur un site atelier sera décrite et les résultats de l'analyse statistique utilisant l'information mutuelle entre les variables seront analysés, en qualifiant la variabilité spatio-temporelle de la couverture végétale en hiver. 


\section{Les facteurs de changement de la couverture hivernale des sols}

6 Les changements intervenant dans la couverture hivernale des sols dans des régions d'agriculture intensive, à forte variabilité spatio-temporelle, dépendent de facteurs de nature socio-économique, politique, physique, etc., qui doivent être définis selon une échelle spatiale appropriée qui permette de les prendre en compte dans une approche environnementale (Thenail et Baudry, à paraître).

\section{Niveaux scalaires des changements}

7 Pour l'étude des changements d'occupation du sol dans les régions où la problématique environnementale est importante, trois niveaux scalaires peuvent être définis (fig. 1).

Figure 1 : Échelles spatiales et facteurs de changements de l'occupation du sol Relation between the spatial scales and the factors of land cover changes

\begin{tabular}{|l|l|}
\hline UNITES SPATIALES & FACTEURS DE CHANGEMENT \\
\hline Bassin versant & $\begin{array}{l}\text { - Actions environnementales } \\
\text { - Facteurs physiques (climat, } \\
\text { topographie, sols) }\end{array}$ \\
\hline Exploitation & $\begin{array}{l}\text { - Variables socio-économiques (statut, } \\
\text { primes) } \\
\text { - Pentes, sols, etc. } \\
\text { - Variables techniques (gestion des } \\
\text { prairies) }\end{array}$ \\
\hline Parcelle agricole &
\end{tabular}

1. À l'échelle $d u$ bassin versant, les mesures environnementales prises par différentes institutions (la Région, le Département, les syndicats de bassins versants, les associations, etc.) sont les principaux facteurs qui interviennent dans les changements d'occupation des sols. Cependant, des conditions climatiques exceptionnelles (inondation ou sécheresse) peuvent également avoir un impact sur la gestion de la couverture hivernale des sols.

2. À l'échelle de l'exploitation, la prise en compte de données socio-économiques (primes, statut et âge de l'exploitant) et physiques (pédologie, morphologie des parcelles, présence d'éléments bocagers) doit permettre d'identifier les facteurs ou variables expliquant les changements d'occupation du sol. À ce niveau d'étude, une base de données importante, mise à disposition par les acteurs locaux, est requise afin de pouvoir spatialiser ces différents facteurs. 
3. À l'échelle parcellaire, le suivi des cycles de successions culturales représente le principal facteur explicatif des changements observés. Les différents systèmes de production répertoriés sont à prendre en compte et à mettre en relation avec des itinéraires techniques et des types de rotations culturales. En Bretagne, plusieurs systèmes de production peuvent être identifiés et rattachés à des rotations "type "; parmi ceux-ci, deux systèmes de production dominants peuvent être cités :

- les systèmes de production « laitier » où l'on retrouve des cycles de rotation intégrant des céréales et des prairies de type temporaire (les surfaces non accessibles par le cheptel sont généralement exclusivement réservées à la culture) :

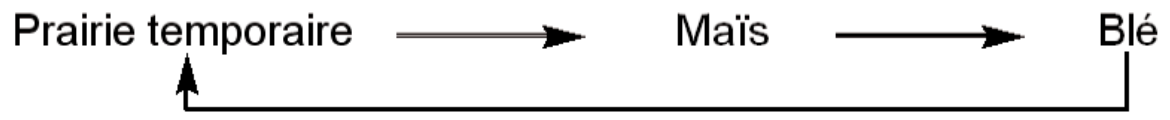

- les systèmes de production hors sol (porcs, volailles) intègrent des rotations avec des successions plus complexes comprenant des protéagineux et une part importante de maïs grain :

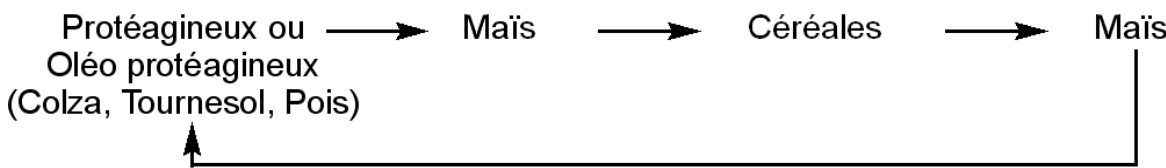

\section{Le rythme des changements}

8 La prise en compte des échelles temporelles dans la gestion de l'occupation hivernale du sol représente également un enjeu important. Des changements observés peuvent ainsi correspondre à des actions qui s'inscrivent dans le court, le moyen ou le long terme (fig. 2). Les projets relatifs à la gestion du paysage tels que des remembrements génèrent par exemple des changements d'occupation hivernale du sol qui ont un impact significatif à long terme sur de grandes superficies. Au contraire, au niveau des pratiques agricoles, une prairie retournée suivi d'un maïs ne sera que très peu de temps potentiellement en sol nu. Une échelle intermédiaire concerne la gestion des successions culturales qui peut s'étaler sur une période allant de 5 à 10 ans. La prise en compte de l'aspect temporel est donc indispensable pour la compréhension des changements observés, une identification des différentes échelles de temps permet ainsi de dégager des tendances d'évolution de l'occupation du sol sur le court et le long terme. 
Figure 2 : Dynamiques temporelles des changements d'occupation du sol pendant l'hiver Time dynamics of the land cover changes during winter

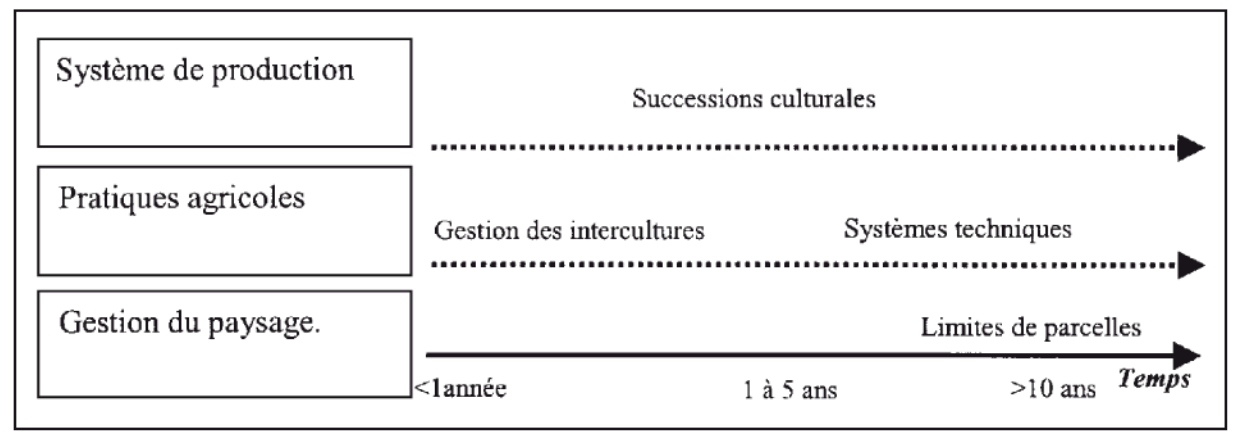

\section{Méthodologie de détection des facteurs de changement de la couverture hivernale des sols sur le bassin versant du Yar}

\section{Présentation de la zone d'étude}

9 Situé à l'extrême nord-ouest du département des Côtes-d'Armor, en limite du département du Finistère, ce bassin versant littoral d'une superficie de $61,5 \mathrm{~km}^{2}$ est à l'origine de plus de $60 \%$ des flux polluants alimentant la baie de Saint-Michel-en-Grève (fig. 3). L'apport important d'éléments nutritifs des cours d'eau entraîne un phénomène d'eutrophisation sur le littoral, couramment appelé « marées vertes » (Esteoule, 1994 ; Molenat et al., 1998). 
Figure 3 : Localisation du bassin versant du Yar Localisation of the Yar drainage bassin

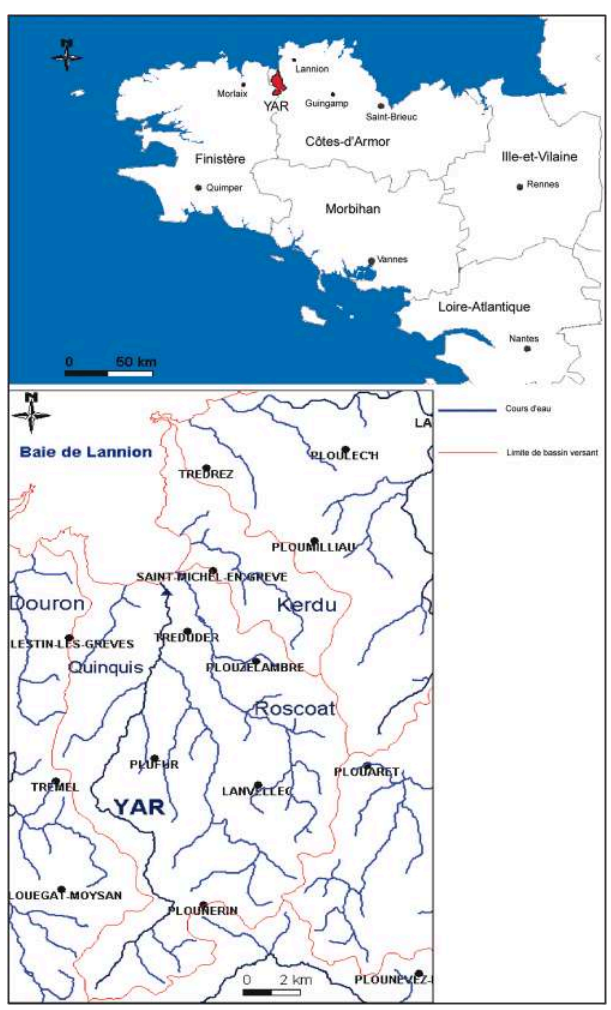

10 À ce titre, il fait l'objet depuis quelques années de nombreuses études de la part de divers organismes de recherche pour tenter de comprendre et d'enrayer le phénomène (CNRS, CEVA, IFREMER, CEMAGREF, INRA ${ }^{1}$ ). Il a notamment été labellisé en 2001 comme site atelier dans le cadre du programme du PEVS (Programme Environnement, Vie et Sociétés du CNRS). Il fait également depuis les années 1990 l'objet de programmes de lutte contre les marées vertes (programme "Algues vertes", puis programme «PROLITTORAL » depuis décembre 2002) initiés notamment par le Conseil régional, l'Agence de l'Eau Loire-Bretagne et les conseils généraux afin de réduire le phénomène d'eutrophisation lié au transfert de nitrates vers les cours d'eau et pour lequel des actions sont engagées par le Comité des bassins versants de la Lieue de Grève, la Chambre d'Agriculture et le Comité professionnel agricole.

De façon générale, le bassin versant du Yar, au paysage bocager assez dense, appartient à une région orientée essentiellement vers l'élevage bovin (Anonyme, 1999) avec une large prédominance de la production lait/viande bovine. La production hors-sol y est surtout de type avicole (uniquement volaille chair), la production porcine étant peu présente sur le bassin versant. On note également la présence de quelques producteurs ovins, d'un maraîcher biologique et d'un céréalier. En moyenne, l'assolement est caractérisé par un système fourrager maïs/herbe (près des trois-quarts de la surface exploitée) avec une large prédominance de l'herbe (près de $50 \%$ de la surface exploitée). La surface moyenne par exploitation est de 49 ha avec un écart type de \pm 31 ha qui traduit la grande diversité des moyens de production qui existe entre les différentes exploitations (moyenne départementale: $39 \mathrm{ha}$ ). Les parcelles situées autour des bâtiments d'élevage sont généralement exploitées en herbe (rotations longues : prairies/maiis/céréales). Les parcelles les plus éloignées sont mises en culture (rotations courtes de type maïs/maïs ou maïs/céréales). L'adaptation à cette contrainte 
par des rotations spécifiques selon l'éloignement du siège de l'exploitation se traduit par une assez forte proportion de sols nus en hiver : $17 \%$ de la SAU en moyenne (Anonyme, 1999).

12 Les programmes d'actions de lutte contre les marées vertes, conjugués avec d'autres programmes visant à la restauration de la qualité de l'eau (PMPOA, Directive Nitrates, etc.) se traduisent par des modifications d'occupation des sols, notamment au travers de changements de modes de production (extensification avec augmentation des prairies, conversion à l'agriculture biologique) et d'une extension de la couverture hivernale des parcelles par des intercultures (la Directive Nitrates, applicable depuis juillet 2001, impose la présence de couverts végétaux pendant l'hiver sur l'ensemble des zones soumises au transfert de nitrates; des actions visant l'implantation d'intercultures ont été mises en place avec le programme "Algue vertes" et sont développées dans le cadre du programme PRO-LITTORAL).

13 Les organismes à l'initiative de ces programmes et/ou de leur application (Conseil général, communauté d'agglomération de Lannion, chambre d'agriculture) se sont montrés intéressés par un outil d'évaluation de leurs actions passées et de programmation pour les actions à venir. L'étude des trajectoires spatio-temporelles de la couverture hivernale des sols contribue à répondre à cette attente.

\section{Détection des classes d'occupation du sol en hiver}

14 La détermination des classes d'occupation du sol en hiver représente une étape déterminante pour la compréhension des facteurs qui motivent les changements. De sa précision dépendra la pertinence du choix des variables expliquant leur évolution temporelle.

15 En Bretagne, la détermination des classes d'occupation des sols en hiver est réalisée à partir du niveau de couverture de végétation des parcelles défini par télédétection. Ainsi, à partir d'une image à haute résolution de type SPOT XS ou $\mathrm{Xi}^{2}$, on distingue les sols couverts, et les sols nus à peu couverts. Ces deux classes sont déterminées à partir d'un indice de végétation, le NDVI (Normalized Difference Vegetation Index), seuillé à l'aide de contrôles effectués sur le terrain, et validé par un croisement avec des données de télédétection acquises a posteriori (Corgne et al., 2002; Lambin et Strahler, 1994). L'utilisation d'une image du printemps ou de l'été suivant permet de déterminer les sols nus "véritables ", c'est-à-dire majoritairement suivis d'un maïs, et de préciser le mode d'occupation des parcelles pendant l'hiver : sols nus précédant un maïs, céréale d'hiver qui à la date de prise de vue est détectée en sol nu grâce à sa faible couverture végétale, intercultures, repousses de végétation spontanées après les récoltes.

Dans un contexte d'étude lié à la restauration de la qualité de l'eau, deux niveaux de précision sont à considérer : l'information sur le degré de couverture du sol en hiver (sol couvert ou sol nu à peu couvert), et la nature de la couverture quand elle existe. Ainsi, une parcelle semée avec des céréales mais qui a un faible niveau de couverture pendant l'hiver joue un rôle non négligeable dans les transferts de flux polluants vers les rivières, mais elle correspond à une parcelle sur laquelle un couvert végétal a été implanté.

17 À partir de la méthode précédemment décrite, deux cartes d'occupation du sol intégrant ces deux niveaux de précision ont été construites en utilisant une série d'images couvrant 7 hivers, à l'exception de l'hiver 2002-2003 pour lequel seul le niveau 
de couverture a été déterminé. L'identification des occurrences des sols nus et des rotations culturales a été effectuée à l'échelle parcellaire sur l'ensemble du bassin versant. Elle a permis de localiser notamment les parcelles caractérisées par des rotations intégrant régulièrement une culture de maïs, et la présence de sols nus « véritables » l'hiver.

Ces cartes fournissent des clés pertinentes dans la compréhension des facteurs déterminant les changements de couverture des sols.

\section{Caractérisation et validation des facteurs déterminant les changements d'occupation du sol}

19 La caractérisation des facteurs qui conditionnent les changements d'occupation du sol nécessite un suivi de l'évolution de l'occupation du sol sur un pas de temps relativement long. Dans cette étude, le suivi réalisé sur sept hivers permet de déterminer sur cette période la répartition spatiale des occurrences de sols nus à peu couverts (fig. 4) et de discriminer ensuite des changements accidentels (liés par exemple à des incidents climatiques) et des changements dépendant des rotations culturales.

Figure 4 : Occurence des sols nus à peu couverts sur le bassin versant du Yar entre 1996 et 2002 Bare soils successions on the drainage bassin of the Yar between 1996 and 2002

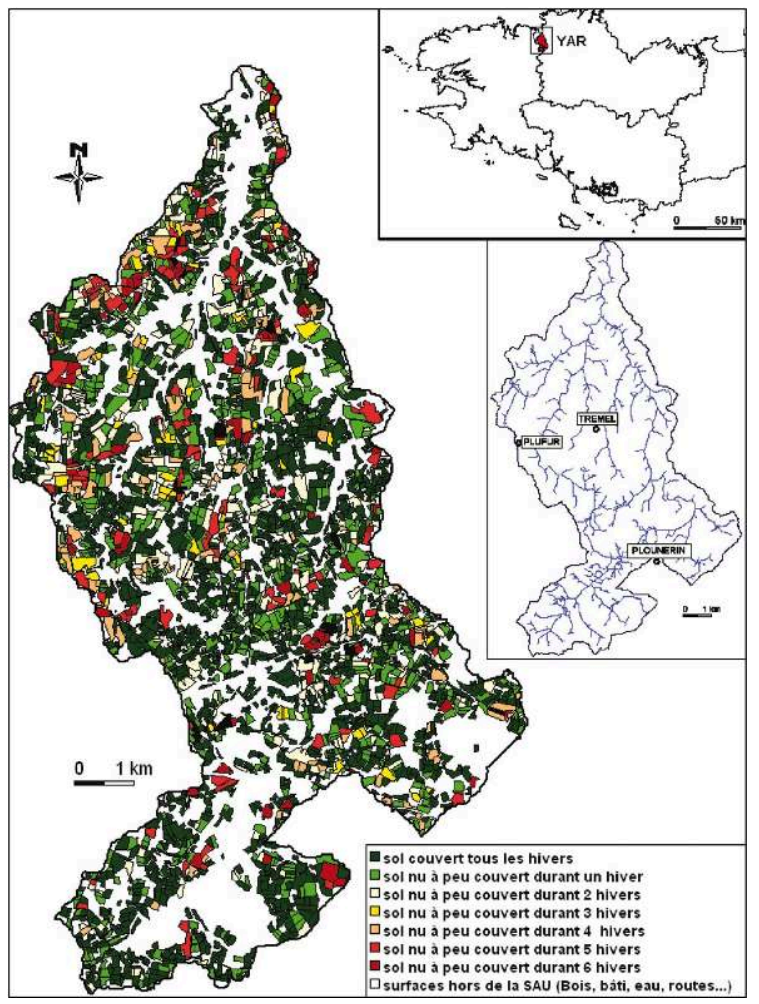

À partir de la base de données constituée par les changements observés, une caractérisation des facteurs déterminant les changements de couverture des sols peut être réalisée. Une consultation préalable des experts en agriculture (agronomes de la chambre d'agriculture des Côtes d'Armor, animateurs de bassin versant) permet, par système de production, de déterminer les facteurs prépondérants expliquant les 
dynamiques d'évolution du sol. Ainsi, sont identifiés pour les systèmes de production de type bovin mixte quatre facteurs : la taille des parcelles; la proximité des parcelles par rapport au siège d'exploitation; les successions culturales; les mesures agroenvironnementales.

Une première validation du choix de ces facteurs a été réalisée au cours d'une étude précédente par un calcul des corrélations entre chacun de ces différents facteurs et la présence de couvert végétal en hiver observé à partir de données de télédétection (Corgne et al., 2002). Pour l'hiver 2002-2003, la figure 5 montre les relations entre les facteurs explicatifs et la variable expliquée qui ont ainsi été mises en évidence (seule la SAU est ici prise en compte; le jeu de classe est binaire : sol couvert et sol nu à peu couvert). L'analyse de relation entre le pourcentage de sols nus en hiver et la variable explicative "occurrence sols nus" a montré que, globalement, plus les occurrences sont importantes, plus la probabilité de retrouver un sol nu à peu couvert pour l'hiver 2002-2003 est forte. La taille des parcelles influe également sur la présence des sols nus hivernaux, puisque les parcelles inférieures à 2 ha sont très majoritairement $(80 \%)$ couvertes par la végétation au cours de l'hiver 2002-2003. La distance de la parcelle au siège d'exploitation joue également un rôle puisque les sols sont plus fréquemment couverts lorsque la distance est élevée. Enfin, comme on pouvait s'y attendre, le choix du mode de production modifie la couverture hivernale des sols, les producteurs en mode biologique implantant davantage d'intercultures que les autres.

Figure 5 : Variations de la présence de couvert végétal en hiver selon les variables explicatives désignées par les experts (analyse réalisée pour l'hiver 2002-2003)

Variations of the land cover allocation in winter according to the explicative variables produced by expert knowledge (study realised for the winter 2002-2003)

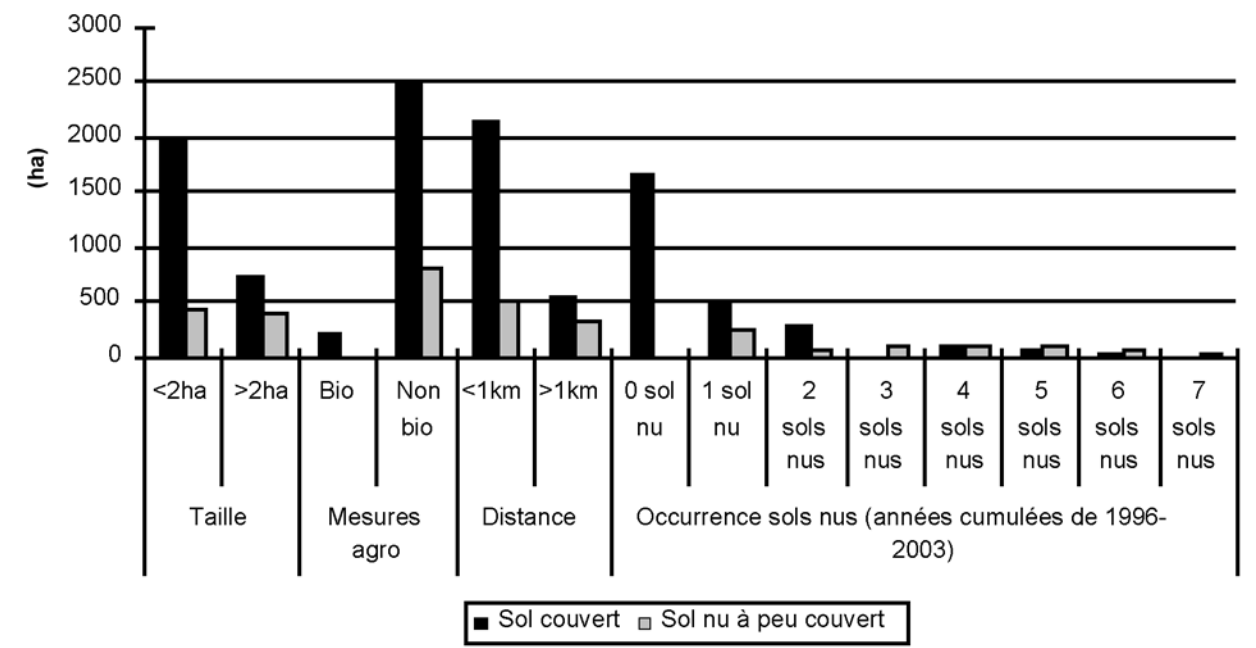

Les facteurs de changement désignés par les experts agricoles ont donc bien une corrélation avec la couverture hivernale des sols. Cependant, cette première validation n'a pas permis de mesurer le "poids» de chaque variable explicative. Or, une hiérarchisation de ces facteurs reste indispensable dans le processus de modélisation de l'occupation hivernale du sol. Une analyse plus fine des données doit ainsi être engagée pour fournir une hiérarchisation complète des variables explicatives. 


\section{La hiérarchisation des facteurs par l'utilisation de l'information mutuelle}

De nombreux outils statistiques existent pour mettre en relation les variables explicatives et la variable à expliquer (analyse factorielle des correspondances, régressions multiples, etc.). Une méthode basée ici sur l'information mutuelle et la redondance entre les variables est appliquée pour hiérarchiser les facteurs identifiés. Cette analyse est basée sur la théorie de l'information (Shanon et Weawer, 1949), utilisée pour quantifier l'information relative à un événement en relation avec l'information apportée par la réalisation de cet événement. Ainsi, la quantité d'information (relative à un contenu statistique) est d'autant plus importante que la réalisation d'un événement est peu probable. Cette théorie a de nombreuses fois été utilisée pour des applications environnementales (Thenail, 2002) et offre l'avantage de travailler sur des valeurs catégorielles, sans contraintes d'effectifs.

Le test d'information mutuelle s'effectue en plusieurs étapes et génère ainsi différents indicateurs expliquant les relations entre les variables (Baudry et Al Jallad, 1992).

1. L'entropie

L'information mutuelle entre les variables est basée sur la notion d'information dont la principale propriété repose sur le fait que la quantité d'information est maximale quand les événements sont équiprobables. Elle permet de calculer les quantités d'informations (entropie, I) entre différentes variables :

$$
I=-\sum_{i=1}^{n} p_{i} \log p_{i} \quad \begin{aligned}
& \mathrm{n}=\text { nombre de résultats possibles } \\
& \mathrm{Pi}=\text { probabilité d'occurrence du résultat } 1
\end{aligned}
$$

\section{L'information mutuelle}

L'information mutuelle entre deux variables, appelée également néguentropie $(\mathrm{N})$, représente l'information apportée par une variable lorsque la seconde est réalisée. Elle est obtenue à partir des calculs d'entropie (I) :

Soit A et B deux variables avec n modalités, alors N (A, B $=$ I (A) + I (B) - I (A, B)*

* I (A, B) représente l'hétérogénéité de la matrice A x B

3. La redondance

La redondance découle des deux premières, elle mesure le taux d'hétérogénéité de deux variables :

$$
\mathrm{R}=\frac{N(A, B)}{I(B)} \text { (ici, on mesure le taux d'hétérogénéité de } \mathrm{B} \text { lié à l'hétérogénéité de } \mathrm{A} \text { ). }
$$

4. Test de signification (K)

Enfin, un test de signification statistique de la liaison entre A et B mesuré par $\mathrm{N}$ ou R peut être calculé avec le critère de Kullback (1959). Celui-ci correspond approximativement à une loi du c2.

La procédure mise en œuvre fournit une hiérarchisation de la quantité d'information des variables explicatives par rapport à la variable à expliquer. Le logiciel « zephyr ", développé à l'INRA (Baudry et Al Jallad, 1992) est ici utilisé pour cette analyse. Les paramètres d'entrée doivent être catégoriels et correspondre à un identifiant de parcelle. 


\section{Résultats}

\section{La prépondérance des occurrences de sols nus à peu couverts observées comme facteur explicatif de l'occupation du sol}

Une analyse statistique basée sur l'information mutuelle fournit de nombreux indicateurs de corrélation entre les variables explicatives et la variable expliquée. Pour cette étude, nous nous intéressons tout d'abord à la néguentropie pour chaque couple de variables et à la redondance qui mesure le taux d'occurrence entre la variable explicative en liaison avec les effectifs de la variable à expliquer. Puis nous nous attacherons à détailler l'entropie des classes de la variable la plus significative selon leur variabilité spatio-temporelle.

\section{Relations entre les variables explicatives et la variable à expliquer}

Les résultats présentent une très forte relation de la variable « occurrence des sols nus à peu couverts » (1996-2002) avec la variable "présence du couvert hivernal pour l'hiver 2002-2003», et une relation beaucoup moins étroite avec les trois autres variables explicatives (tableau 1).

Tableau 1 : Hiérarchisation des variables explicatives à partir de l'analyse par information mutuelle Hierarchization of the explicative variables with the mutual information analysis

\begin{tabular}{|c|c|c|c|c|c|c|}
\hline $\begin{array}{l}\text { Variables } \\
\text { explicatives }\end{array}$ & Classes & $\begin{array}{c}\text { Effectifs } \\
\text { (nombre de } \\
\text { parcelles et } \\
\text { pourceutage) }\end{array}$ & $\begin{array}{c}\text { Kedon- } \\
\text { dance }\end{array}$ & $\begin{array}{l}\text { Entropie } \\
\text { mutuelle }\end{array}$ & $\begin{array}{c}\text { Information } \\
\text { mutuelle } \\
\text { (Néguentropie) }\end{array}$ & $\begin{array}{l}\text { Test de } \\
\text { Signifi- } \\
\text { cation }\end{array}$ \\
\hline \multirow{2}{*}{$\begin{array}{c}\text { Proximité de la } \\
\text { parcelle par } \\
\text { rapport au siègc } \\
\text { d'exploitation } \\
\end{array}$} & $\begin{array}{l}1: \text { parecllc }<1 \mathrm{Km} \text { du } \\
\text { siége d'exploitation }\end{array}$ & $3082(80,6 \%)$ & \multirow{2}{*}{$2,30 \%$} & \multirow{2}{*}{0,902} & \multirow{2}{*}{0.100} & \multirow{2}{*}{75,55} \\
\hline & $\begin{array}{l}2: \text { parcelic }>1 \mathrm{Km} \mathrm{du} \\
\text { siège d'exploitation }\end{array}$ & $697(18,4 \%)$ & & & & \\
\hline \multirow[b]{2}{*}{$\begin{array}{c}\text { Mcsurc agro } \\
\text { enviroumementale }\end{array}$} & $\begin{array}{l}\text { 1: parcelle concernée } \\
\text { par une mesure agro } \\
\text { environnementale }\end{array}$ & $196(5,19 \%)$ & \multirow[b]{2}{*}{$0,23 \%$} & \multirow[b]{2}{*}{0,637} & \multirow[b]{2}{*}{0,0010} & \multirow[b]{2}{*}{1,94} \\
\hline & $\begin{array}{c}2 \text { : parcelle non } \\
\text { concernée par une } \\
\text { mesure agro } \\
\text { environnementale }\end{array}$ & $3583(94,8 \%)$ & & & & \\
\hline \multirow{2}{*}{$\begin{array}{l}\text { Taille des } \\
\text { parcelles }\end{array}$} & 1: parcelle $<2$ ha & $3413(90,3 \%)$ & \multirow{2}{*}{$2,62 \%$} & \multirow{2}{*}{0,741} & \multirow{2}{*}{0,114} & \multirow{2}{*}{22,41} \\
\hline & 2 : parcelle > 2 ha & $366(9,7 \%)$ & & & & \\
\hline \multirow{7}{*}{$\begin{array}{l}\text { Occurrences des } \\
\text { sols nus à peu } \\
\text { couverts } \\
(1996-2002)\end{array}$} & $\begin{array}{c}1: \text { sol couvert tous les } \\
\text { hivers }\end{array}$ & $2185(57,8 \%)$ & \multirow{7}{*}{$44,25 \%$} & \multirow{7}{*}{1,547} & \multirow{7}{*}{0,192} & \multirow{7}{*}{103,2} \\
\hline & $\begin{array}{l}2 \text { : sol nu à peu couvert } \\
\text { durant un hiver }\end{array}$ & $770(20,4 \%)$ & & & & \\
\hline & $\begin{array}{l}3: \text { sol nu à pell couvert } \\
\text { durant } 2 \text { hivers }\end{array}$ & $352(9,31 \%)$ & & & & \\
\hline & $\begin{array}{c}4: \text { sol nu à peu couvert } \\
\text { durant } 3 \text { hivers }\end{array}$ & $85(2,25 \%)$ & & & & \\
\hline & $\begin{array}{l}5: \text { sol trul d pelt couvert } \\
\text { durant } 4 \text { hivers }\end{array}$ & $159(4,21 \%)$ & & & & \\
\hline & $\begin{array}{c}6: \text { sol nu à peu couvert } \\
\text { durant } 5 \text { hivers }\end{array}$ & $118(3,12 \%)$ & & & & \\
\hline & $\begin{array}{l}7: \text { sol nu à peu couvert } \\
\text { durant } 6 \text { hivers }\end{array}$ & $110(2,91 \%)$ & & & & \\
\hline
\end{tabular}

La néguentropie, qui mesure la connaissance que l'on a de la variable expliquée quand on connait la variable explicative, illustre la forte relation de la variable « occurrence des sols nus à peu couverts» (1996-2002) avec la variable "présence du couvert hivernal pour l'hiver 2002-2003» $(0,192)$. Au contraire, pour la variable « mesures agroenvironnementales », on obtient 0,001 ce qui signifie que tous les états de la variable 
" présence du couvert hivernal pour l'hiver 2002-2003 » sont équiprobables quelles que soient les modalités de la variable explicative. Un si faible résultat s'explique d'une part par le faible effectif des parcelles concernées par des mesures environnementales $(5,19 \%)$, et d'autre part, par le fait que dans cette variable sont intégrés les CTE (contrats territoriaux d'exploitation) et les conversions biologiques, caractérisés chacun par des modes de gestion spécifiques. Les variables " proximité » et « taille des parcelles » ont des valeurs sensiblement identiques plutôt faibles, indiquant cependant une relation significative avec la variable à expliquer.

La redondance, qui mesure l'organisation du système, montre ainsi que 44,25\% des modalités de la variable "présence du couvert hivernal pour l'hiver 2002-2003 » sont liées à la variable "occurrence des sols nus à peu couverts (1996-2002)». Viennent ensuite la variable «taille des parcelles" avec $2,62 \%$, la variable "proximité de la parcelle au siège d'exploitation" avec $2,30 \%$ et la variable "mesures agroenvironnementale» avec $0,23 \%$. On note également que toutes les variables explicatives ont un degré de signification supérieure à $5 \%$ ce qui prouve une relation effective, statistiquement significative, entre toutes les variables explicatives et la variable expliquée.

\section{Analyse de l'entropie de la variable " occurrence des sols nus à peu couverts »}

La distribution spatiale de l'entropie entre les différentes classes de la variable « occurrence des sols nus à peu couverts » en relation avec la variable «présence du couvert hivernal pour l'hiver 2002-2003" est illustrée par la figure 6. Une forte corrélation spatiale entre la figure 4 et la figure 6 traduit le poids élevé de la variable "occurrences des sols nus à peu couverts" dans l'explication de la couverture hivernale des sols pour l'hiver 2002-2003. L'entropie étant maximale quand tous les événements sont équiprobables, lorsque celle-ci est égale ou proche de zéro, une des classes de la variable est alors fortement corrélée à la variable expliquée. C'est le cas ici pour les parcelles en sol couvert tous les hivers et pour les parcelles en sols nus à peu couverts durant 3 hivers. Pour ces dernières, la totalité des parcelles ( 85 parcelles) est en sol nu à peu couvert au cours de l'hiver 2002-2003. Pour les parcelles couvertes tous les hivers, qui correspondent généralement à des prairies permanentes, 99,8 \% d'entre elles sont détectées en sol couvert pour l'hiver 2002-2003, ce qui explique la très faible entropie mesurée $(0,013)$ et montre la très forte stabilité spatio-temporelle de cette classe. Elle couvre en moyenne sur la période d'observation $35 \%$ de la SAU, la place des prairies étant importante dans les systèmes de production présents sur le bassin versant du Yar. Les parcelles concernées par de fortes occurrences de sols nus à peu couverts (4 à 6 sur la période étudiée) sont a contrario caractérisées par une forte entropie $(0,599$ à 0,688$)$. Par exemple, sur les 159 parcelles caractérisées par 5 hivers en sols nus à peu couverts sur la période étudiée, 93 sont en sol couvert en 2002-2003 et 66 en sols nus à peu couverts. Ces parcelles sont donc caractérisées par une très forte variabilité d'évolution de l'occupation hivernale du sol. 
Figure 6 : Enthropie entre les classes de la variable « Occurrences des sols nus à peu couverts » et la variable "Présence du couvert hivernal pour l'hiver 2002-2003 » sur la bassin versant du Yar Entropy between the classes of the variable "Bare soil successions" and the variable "Land cover allocation for the winter 2002-2003" on the drainage bassin of the Yar

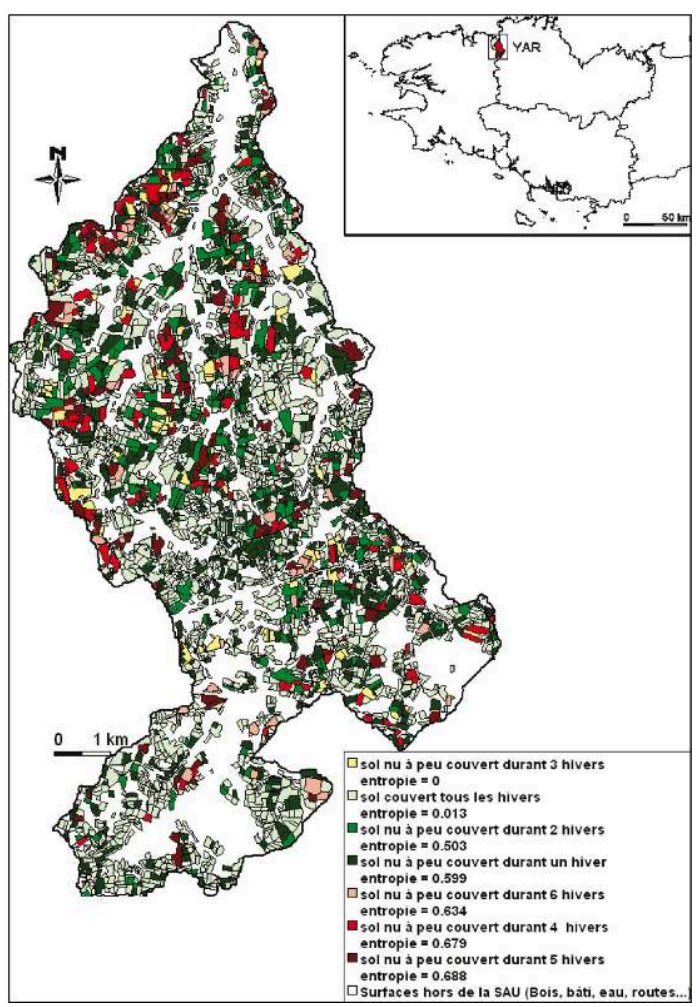

\section{Les facteurs explicatifs des « occurrences des sols nus à peu couverts $\gg$ observés sur la période 1996-2003}

Après avoir hiérarchisé les variables explicatives, une classification par hiérarchie descendante est effectuée à partir de la variable "occurrences des sols nus (1996-2003) » afin de déterminer, pour chacun des modes ou classe de cette variable, des combinaisons hiérarchiques des trois autres variables qui ont un poids plus faible dans l'explication de la présence du couvert végétal au cours de l'hiver 2002-2003. La procédure consiste donc à identifier des « groupes de solutions » construits à partir des modalités de la variable fournissant le plus d'information (fig. 7). Trois règles d'arrêt sont intégrées dans la procédure de classification : celle-ci est stoppée lorsqu'il n'y a plus assez de parcelles (le seuil est ici fixé à 10), ou lorsque l'entropie est trop faible ou encore lorsque toutes les variables explicatives sont utilisées. Dans cette étude, 10 groupes de solution sont créés d'après ces règles d'arrêt. 
Figure 7 : Étapes de la création des " groupes de solutions » par classification descendante Stages of the determination of the "group solutions" with tree classification

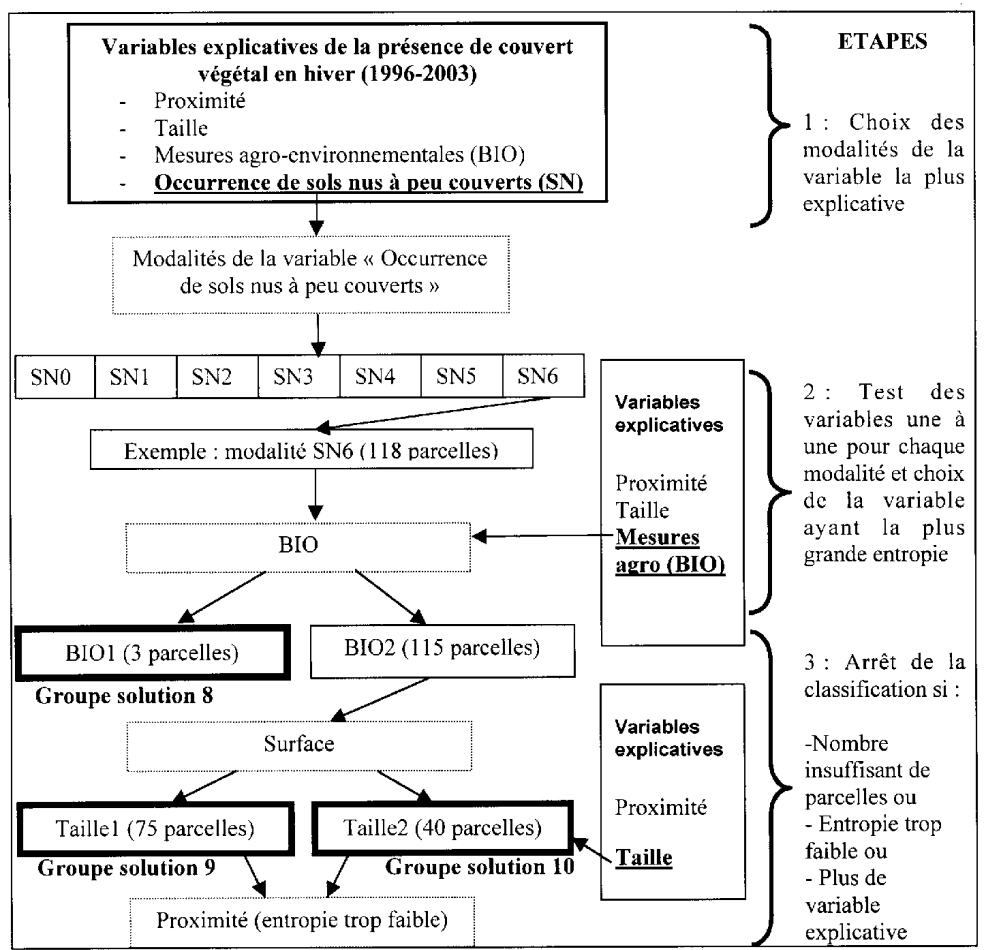

31 Chaque "groupe de solutions" est créé à partir des 7 modalités de la variable « occurrences des sols nus à peu couverts (1996-2003)», chacune de ces modalités ou classes, de « SN0 » à « SN6 » correspondant à la fréquence de retour de sol nu sur la parcelle au cours des 7 hivers.

Un exemple est donné dans la figure 5 avec la modalité SN6 (qui correspond aux parcelles détectés 6 hivers en sols nus à peu couverts). Celle-ci concerne 118 parcelles dont la relation la plus forte se fait avec la variable «mesure agro-environnementale ». La classification s'arrête alors pour la modalité BIO1 (parcelles concernées par des mesures agro-environnementales) puisque le nombre de parcelles est insuffisant (Groupe solution 8). Elle continue par contre avec la modalité BIO2 (parcelles sans mesures agro-environnementales) fortement liée à la variable Taille composée de deux modalités : Taille1 (inférieur à 2 ha) et Taille2 (supérieure à 2 ha). L'entropie avec les modalités de la dernière variable Distance étant trop faible, la classification est stoppée et les groupes de solutions identifiés (Groupe solution 9 et 10).

Sur les 10 groupes solutions identifiés, 7 sont caractérisés dès la $1^{\text {re }}$ étape car l'entropie avec les autres variables est trop faible, ainsi, seuls 3 groupes de solution sont construits en relation avec les autres variables et comme pour l'exemple exposé pour la modalité SN6, les variables «mesure agro-environnementale » et «taille » présentent le plus de corrélation.

34 Néanmoins, l'étude des différents groupes en relation avec les 3 variables explicatives s'avère intéressante. Ainsi, l'analyse du quatrième groupe, calculée à partir de la quatrième modalité de la variable «occurrence des sols nus à peu couverts (SN4) » montre que sur les 85 parcelles composant ce groupe de solutions, la totalité est en sols nus à peu couverts au cours de l'hiver 2002-2003, mais seulement 28 d'entre elles sont 
localisées à une distance supérieure à $1 \mathrm{~km}$ de l'exploitation et un quart de ces parcelles ont une surface supérieure à 2 ha.

Pour palier les faibles valeurs informatives des variables " proximité », « mesure agroenvironnementale » et «taille des parcelles », une structuration plus fine des données d'entrée devrait permettre de ne pas sous évaluer le poids de ces variables : ainsi, les faibles niveaux de redondance obtenus pour la variable "proximité » s'expliquent entre autres par le fait que de nombreuses parcelles ont leur siège d'exploitation localisé à l'extérieur du bassin versant ; par ailleurs des groupements d'exploitation ont pu avoir lieu, rendant le jeu de données obsolète. Enfin, une description plus détaillée des types de mesures environnementales concernant chaque exploitation fournirait pour cette variable un apport informatif important dans sa relation avec la couverture hivernale des sols.

\section{Conclusion}

L'identification, la structuration et la hiérarchisation des facteurs de changement d'occupation du sol dans les régions d'agriculture intensive représentent un enjeu important dans la compréhension des dynamiques spatiales ayant un impact sur l'environnement. Ce travail avait pour objectif de hiérarchiser les facteurs déterminant les changements de l'occupation hivernale du sol sur un bassin versant faisant l'objet d'un programme de reconquête de la qualité de l'eau.

Une analyse basée sur l'information mutuelle a permis de hiérarchiser les différentes variables expliquant les changements intervenus entre deux hivers successifs et exprimées à dire d'expert. La variable "occurrences des sols nus à peu couverts", déterminée à partir de données satellitaires, a été identifiée comme étant le facteur explicatif dominant parmi les différentes variables identifiées pour expliquer la présence du couvert végétal sur les parcelles pour l'hiver 2002-2003, soulignant ainsi le faible poids des autres variables qui ne fournissent que peu d'informations sur la variable à expliquer. Cette variable, intégrant les dimensions spatiales et le temporelles de l'évolution du couvert végétal, s'inscrit dans une dynamique pluri-annuelle des successions culturales où une relation de type logique apparaît entre le nombre d'occurrence de sols nus à peu couverts observés dans les hivers précédents et l'état de l'occupation du sol au cours de l'hiver 2002-2003. Le poids très élevé de cette variable traduit une reproduction du système observé sur la période 1996-2000. Ceci s'explique par le fait que les effets liés à la Directive Nitrates ne sont pas encore lisibles, l'application effective de cette directive sur le terrain se produisant avec une ou deux années de décalage, sans compter l'effet des variations climatiques sur l'implantation et le développement du couvert hivernal. Le poids de la variable "mesure agrienvironnementale » devrait être p<lus élevé dès l'hiver 2003-2004, d'autant que les mesures mises en place dans le cadre du programme " PRO-LITTORAL", à travers les contrats d'objectifs passés depuis 2003 entre les agriculteurs et le Comité des bassins versants de la Lieue de Grève, favorisent l'implantation des intercultures.

Une analyse comparative des différentes variables a été conduite à partir d'une classification hiérarchique descendante des données, qui crée des groupes de solutions calculés à partir des modalités de la variable la plus significative, l'analyse de ces groupes autorisant ainsi la comparaison des variables. On constate alors que mise à part la variable " occurrence des sols nus à peu couverts », caractérisée par un test de 
signification important, les trois autres variables, bien que significatives, n'apportent pas l'information escomptée dans l'explication des changements d'occupation du sol. Cette analyse a également montré la nécessité d'une structuration plus fine des données d'entrée afin de ne pas sous-estimer le poids de certaines variables dans l'explication de la présence du couvert hivernal des sols.

\section{BIBLIOGRAPHIE}

BAUdRY (J.), AL JALLAD (A.), 1992. - « La théorie de l'information : un outil d'analyse des liaisons entre les variables », Rapport CERESA, INRA, Rennes, 8 p.

ANONYME, 1999. - «Lutte préventive et curative contre la prolifération des marées vertes, Conception d'un programme d'actions préventives en Baie de Lannion ", Rapport SCE/FEDR/CG 22, BEP II, Saint-Brieuc, Conseil général des Côtes-d'Armor, 87 p.

CORgne (S.), Hubert-moy (L.), Barbier (J.), Solaiman (B.), 2002. - « Follow-up and modeling of the land use in an intensive agricultural watershed in France ", Proceedings of SPIE, vol. 4879, p. 342-351.

DABney (S. M.), 1998. - « Cover crop impacts on watershed hydrology », J. Soil Water Cons., 53, p. 207-213.

ESTEOULE (J.), 1994. - «Étude des transferts de nutriments alimentant l'eutrophisation en milieu continental et littoral sur le bassin versant du Yar en baie de Lannion ", Rapport du Centre National du Machinisme Agricole du Génie Rural des Eaux et des Forêts, Rennes, 48 p.

Hubert-Moy (L.), Cotonnec (A.), Le Du (L.), Chardin (A.), Perez (P.), 2001. - « A comparison of classification procedures of remotely sensed data applied on different landscape units ", Remote Sensing of Environment, vol. 75, $\mathrm{n}^{\circ}$ 2, p. 174-187.

Hubert-Moy (L.), Corgne (S.), Mercier (G.), Solaiman (B.), 2002. - « Land use and land cover change prediction with the theory of evidence : a study case in an intensive agricultural region in France ", Fusion 2002 Conference, ISIF IEEE, p. 114-122.

JAUNATRE (D.), 1997. - «Influence des techniques culturales sur le ruisselllement et les transferts de polluants à l'échelle d'un versant en contexte armoricain ", mémoire de fin d'études École Supérieure d'Ingénieurs et de Techniciens pour l'Agriculture, Rennes, $57 \mathrm{p}$.

KULlBACK (S.), 1959. - «Information theory and statistics », John Wiley and Sons, New York, 395 p.

LAMBIN (E. F.), STRAHLER (A. H.), 1994. - « Indicators of land cover change for change vectors analysis in multitemporal space at coarse spatial scale », International Journal of Remote Sensing, vol. $15, \mathrm{n}^{\circ} 10$, p. 2099-2119.

Molenat (J.), Gascuel-Odoux (C.), Merot (Ph.), Durand (P.), 1998. - « Diagnostic approfondi sur le Yar et le Roscoat : approche de la circulation de l'eau au sein des bassins versants », Rapport de l'INRA, Rennes, $45 \mathrm{p}$.

SHANON (C. E.), WEAVER (W.), 1949. - « The mathematical theory of communication », Chicago, University of Illinois Press, $144 \mathrm{p}$. 
THENAIL (C.), 2002. - « Relationships between farm characteristics and the variation of the density of hedgerows at the level of a micro-region of bocage landscape. A study case in Brittany, France ", Agricultural Systems, 71, p. 207-230.

THENAIL (C.), BAUDRY (J.), (à paraître). - « Variation of the spatial land use pattern in farms according to the structure of the hedgerow network landscape. A case study in northeast Brittany, France ", Agricultural System.

WICHEREK (S.), 1996. - « Impact de l'agriculture sur la qualité des sols et des eaux », dans GAUDEMAR (J.-P.), Environnement et Aménagement du territoire, Paris, Documentation Française, p. 117-124.

\section{NOTES}

1. CNRS : Centre National de la Recherche Scientifique ; CEVA : Centre d'Étude et de Valorisation des Algues; IFREMER : Institut Français pour l'Étude et l'Exploitation de la Mer; CEMAGREF : Centre National du Machinisme agricole, du Génie rural des Eaux et des Forêts; INRA : Institut National de la Recherche Agronomique.

2. Satellite multi spectral comprenant 3 bandes pour SPOT XS (vert, rouge et Proche Infrarouge) et 4 bandes pour Xi (vert, rouge, Proche Infrarouge et Moyen Infrarouge) de 20 mètres de résolution spatiale.

\section{RÉSUMÉS}

La Bretagne connaît depuis plusieurs décennies des changements de systèmes culturaux, liés à des modifications des structures paysagères et des pratiques agricoles entraînant des problèmes environnementaux, en particulier une dégradation importante des ressources en eau. Dans ce contexte, la connaissance des variations spatio-temporelles de l'utilisation et de l'occupation des sols constitue un élément-clé dans une démarche de restauration de la qualité de l'eau. L'objectif de ce travail est de définir le poids respectif des facteurs de changements identifiés en hiver sur un bassin versant localisé en secteur agricole assez intensif. Ainsi, quatre facteurs sont identifiés pour les systèmes de production de type bovin mixte : la taille des parcelles; la proximité des parcelles par rapport au siège d'exploitation; les occurrences des sols nus à peu couverts; les mesures agro-environnementales.

Une analyse statistique utilisant l'information mutuelle est ensuite appliquée afin de hiérarchiser les différentes variables explicatives exprimées à dire d'expert et validées à travers une analyse statistique. On constate alors que mise à part la variable «occurrence des sols nus à peu couverts", définie à partir d'une série d'images satellitaires et caractérisée par un test de signification important, les trois autres variables, bien que significatives, n'apportent pas l'information escomptée dans l'explication les dynamiques pluri-annuelles d'occupation des sols. Il apparaît ainsi nécessaire de structurer plus finement les variables explicatives en entrée afin de ne pas sous-estimer leur poids dans l'explication de la présence du couvert hivernal des sols.

Changes in farming systems occur for several decades in Brittany, related with changes of both landscape structure and agricultural practices that lead to environmental problems, especially a serious degradation of water quality. 
In this context, knowledge of the spatio-temporal variations of land cover changes represents a key indicator for the restoration of the water quality. The aim of this paper is to define the weight of the driving factors of land cover change in winter in a quite intensive agricultural region. Four driven factors are identified for the dairy-cattle farming: Proximity of the fields to the farm building, environmental actions, bare soil occurrence.

A statistical analysis based on the mutual information is used to hierarchize the explicative variables defined from expert knowledge and statistically validated. Results highlight the high contribution of the variable "bare soil occurrence", which is built with a series of satellite images and characterized with an important signification test. The other driving factors can be seen as somewhat significant, although they don't provide the expected information allowing to explain pluri-annual dynamics of land cover. Therefore, it appears necessary to restructure the input parameters of these explicative variables to not underestimate their influence on land cover change in winter.

INDEX

Index géographique : France, Bretagne

Keywords : land cover, remote sensing

Mots-clés : occupation du sol, télédétection

\section{AUTEUR}

SAMUEL CORGNE

COSTEL - Université Rennes 2 Haute-Bretagne, LETG-UMR 6554/CAREN IFR 90 - CNRS, samuel.corgne@uhb.fr 